\title{
Domains of attraction of interconnected systems: A Zubov method approach
}

Fabio Camilli
Lars Grüne

Fabian Wirth

\begin{abstract}
We study feedback interconnections of two nonlinear systems, that are asymptotically stable at a fixed point. It is shown that if the subsystems are input-to-state stable and the corresponding gains satisfy a small gain condition, then estimates for the domain of attraction of the whole system may be obtained by calculating robust Lyapunov functions for the subsystems. The latter task can be solved using available Zubov techniques. In total this approach makes numerical computations feasible, as high cost computations only have to be performed in lower dimensions. This comes at the price, that in general only lower approximations of the domain of attraction are obtained and that the system has to be brought into a form, where a small gain condition holds.
\end{abstract}

\section{INTRODUCTION}

In this note we present a method for the estimation of the domain of attraction of asymptotically stable fixed points of higher dimensional systems. Our approach is based on extensions of Zubov's method that were developed in [1], [2], [3]. In this approach a first-order partial differential equation is formulated which has a unique viscosity solution vanishing in the fixed point. This viscosity solution is a maximal Lyapunov function on the robust domain of attraction of the perturbed system. In [1] it has been shown that the resulting first-order PDE may be regularized in such a manner that on the one hand the solution still yields a function characterizing the domain of attraction and so that on the other hand the solution can be accurately computed using suitable numerical methods. In principle this approach is riddled by the curse of dimensionality as the computation of the solution becomes prohibitively expensive in higher dimensions, where "high" actually only means at least starting from dimension 5. To overcome this problem for larger systems we assume that the higher dimensional system is given as an interconnection of lower dimensional ones. To this end it is assumed that the lower dimensional subsystems satisfy an input-to-state stability (ISS) property with respect to the influence of the states of the other subsystems.

We show that ISS Lyapunov functions may be calculated for the individual subsystems using a Zubov approach. The method we present here is a direct extension of the methods and results in [1]. An alternative method for the calculation of ISS Lyapunov functions has been presented within the

This work was not supported by any organization

F. Camilli is with the Sezione di Matematica per L'Ingegneria Dipartimento di Matematica Pura ed Applicata Università degli Studi di L'Aquila, Italy camilli@ing. univaq.it

L. Grüne is with the Mathematical Institute, University of Bayreuth, Germany lars.gruene@uni-bayreuth.de

F. Wirth is with the Institute of Mathematics, University of Würzburg, Germany wirth@mathematik.uni-wuerzburg.de framework of input-to-state dynamical stability in [8]. Building on recent advances in the understanding of stability properties of interconnected ISS systems, we show that a Lyapunov function for the overall system can be obtained in a straightforward manner from the Lyapunov functions of the subsystems provided a small gain condition is satisfied. To this end we assume that a small gain condition is satisfied for the gains given by the individual ISS Lyapunov functions. Here we use results obtained in [11], [10], [5], [7], [4], [12].

In this note we restrict our attention to the coupling of two systems in a feedback interconnection. However, as the tools from viscosity theory that we require are only needed in the analysis of individual subsystems and as the theory of interconnection of ISS systems has been developed in a general framework, we expect that these methods will extend in a straightforward manner to more general interconnection structures. This will be the topic of further articles which are under preparation.

We proceed as follows. In the ensuing Section II we present the problem description and recall the relevant stability definitions used in the paper. The key in the analysis of the subsystems is the introduction of an auxiliary system which we present in Section III. For the auxiliary system a robust Lyapunov function is constructed using Zubov's method in Section IV. These Lyapunov functions serve as ISS Lyapunov functions for the coupled system. In Section V we explain how to obtain estimates for the domain of attraction for the coupled system. An academic example is presented in VI. In Section VII we briefly explain how a certain way of preconditioning the system may transform a given system into a form where the approach of this paper is applicable.

\section{PRELIMINARIES AND PROBLEM STATEMENT}

In this paper the Euclidean norm in $\mathbb{R}^{n}$ is denoted by $\|\cdot\|$ and $B(z, r):=\left\{x \in \mathbb{R}^{n} \mid\|x-z\|<r\right\}$ denotes the set of points with distance less than $r$ from $z$. As usual in stability analysis, we call a function $\alpha$ of class $\mathscr{K}_{\infty}$ if it is a homeomorphism of $[0, \infty)$ (i.e. $\alpha$ is continuous, $\alpha(0)=0$ and $\alpha$ is strictly increasing to infinity). A continuous function $\beta: \mathbb{R}_{+} \rightarrow \mathbb{R}_{+}$ is of class $\mathscr{L}$ if it is strictly decreasing to 0 as $r \rightarrow \infty$; and we call a continuous function $\beta$ with two real nonnegative arguments of class $\mathscr{K} \mathscr{L}$ if it is of class $\mathscr{K}_{\infty}$ in the first and of class $\mathscr{L}$ in the second argument.

In this paper we will study stability properties of coupled systems. To this end we need the concept of input-to-state stability, which was first defined in [13]. We consider a 
system

$$
\dot{x}=f(x, u) \text {. }
$$

where $x \in \mathbb{R}^{n}$ is the state and $u \in \mathbb{R}^{m}$ is the control input. We assume that $f$ is regular enough such that for each initial condition $x^{0} \in \mathbb{R}^{n}$ and each control input $u \in L^{\infty}$ a unique solution exists for all $t \geq 0$. System (II.1) is called input-tostate stable (ISS), if there is a gain $\gamma \in \mathscr{K}_{\infty}$ and a $\beta \in \mathscr{K} \mathscr{L}$ such that

$$
\left\|x\left(t, x^{0}, u\right)\right\| \leq \beta\left(\left\|x^{0}\right\|, t\right)+\gamma\left(\|u\|_{\infty}\right),
$$

for all initial conditions $x^{0} \in \mathbb{R}^{n}, t \geq 0$ and essentially bounded, measurable inputs $u(\cdot) \in L^{\infty}\left(\mathbb{R}_{+}, \mathbb{R}^{m}\right)$.

In this paper we will use the equivalent Lyapunov characterization of input-to-state stability in what is called the implication form.

Definition 1 A smooth function $V$ is called an ISS-Lyapunov function of (II.1) if there exist $\psi_{1}, \psi_{2} \in \mathscr{K}_{\infty}$ and $\chi, \alpha \in \mathscr{K}$ with

$$
\begin{gathered}
\psi_{1}(\|x\|) \leq V(x) \leq \psi_{2}(\|x\|), x \in \mathbb{R}^{n}, \\
V(x) \geq \chi(\|u\|) \Rightarrow \nabla V(x) f(x, u) \leq-\alpha(V(x)) .
\end{gathered}
$$

The function $\chi$ is then called Lyapunov-gain.

It is known that the ISS property of (II.1) is equivalent to the existence of an ISS-Lyapunov function for (II.1), see [15]. But note that the gain in (II.2) and the Lyapunov-gain in (II.4) are generally different functions.

Also note that because of (II.3) the implication (II.4) is implied by

$$
\|x\| \geq \psi_{2}^{-1} \circ \chi(\|u\|) \Rightarrow \nabla V(x) f(x, u) \leq-\alpha(V(x)) .
$$

In the following constructions we will arrive at inequalities of the form (II.5). We also will only arrive at local stability results, so we note that local versions of ISS or its Lyapunov versions are simply defined by requiring (II.2) respectively (II.4) to hold for all $x^{0}$ in a neighborhood of $x^{*}=0$ and all $u \in L^{\infty}$ with a norm bound.

We consider a system of two interconnected systems without control (or perturbation). In the following $n_{1}, n_{2} \in \mathbb{N}$ denote the dimension of the two subsystems and we let $N:=n_{1}+n_{2}$. Given $f_{i}: \mathbb{R}^{N} \rightarrow \mathbb{R}^{n_{i}}, i=1,2$, we consider the system

$$
\left\{\begin{array}{l}
\dot{x}_{1}=f_{1}\left(x_{1}, x_{2}\right) \\
\dot{x}_{2}=f_{2}\left(x_{2}, x_{1}\right) \\
x_{1}(0)=x_{1}^{0}, x_{2}(0)=x_{2}^{0}
\end{array}\right.
$$

We let $f=\left(f_{1}, f_{2}\right): \mathbb{R}^{N} \rightarrow \mathbb{R}^{N}, x=\left(x_{1}, x_{2}\right), x^{0}=\left(x_{1}^{0}, x_{2}^{0}\right)$ so that we may equivalently write the system as

$$
\left\{\begin{array}{l}
\dot{x}=f(x) \\
x(0)=x^{0}
\end{array}\right.
$$

It is assumed that $f$ is locally Lipschitz continuous on $\mathbb{R}^{n}$, has a fixed point in $x^{*}=0$ and that the fixed point is locally exponentially stable. Further $x\left(t, x^{0}\right)$ denotes the solution of (II.7) at time $t$ corresponding to the initial condition $x(0)=$ $x^{0}$. We are interested in the domain of attraction of the fixed point, that is in the set

$$
\mathscr{D}=\left\{x^{0} \in \mathbb{R}^{N}: x\left(t, x^{0}\right) \rightarrow 0 \quad \text { for } t \rightarrow \infty\right\} .
$$

In the following approach the coupling structure into account explicitly. The assumptions so far imply that

$$
f_{1}(0,0)=0, \quad f_{2}(0,0)=0
$$

but they do not imply that the individual subsystems if they are uncoupled are exponentially stable. That is, we have no stability property for the systems

$$
\dot{x_{i}}=f_{i}\left(x_{i}, 0\right) \text {. }
$$

This may be seen even in the linear case by considering the system

$$
\dot{x}=\left(\begin{array}{ll}
1 & -1 \\
4 & -3
\end{array}\right) x, \quad x \in \mathbb{R}^{2} .
$$

The matrix has a double eigenvalue at -1 , so that the system is exponentially stable, however, the first subsystem is not exponentially stable. For nonlinear systems it is not too hard to construct systems which are asymptotically stable at $x^{*}=0$ and so that both of the uncoupled subsystems are not stable at $x_{i}^{*}=0$. We will discuss in Section VII that if this is the case, then it is always possible to transform the system into an equivalent system in which the subsystems have the stability properties we need in the following arguments.

For the moment we introduce the extra assumption that each of the subsystem has nice stability properties, which means in our context that they satisfy an ISS property. In the following $i \in\{1,2\}$ and $i \neq j \in\{1,2\}$ we will be the complementary index.

We assume that for $i=1,2$ the there exist $\gamma_{i j} \in \mathscr{K}_{\infty}$ and $\beta_{i} \in \mathscr{K} \mathscr{L}$ such that

$$
\left\|x_{i}\left(t, x_{i}^{0}, x_{j}(\cdot)\right)\right\| \leq \beta_{i}\left(\left\|x_{i}^{0}\right\|, t\right)+\gamma_{i j}\left(\left\|x_{j}\right\|_{\infty}\right),
$$

for all $x_{i}^{0} \in \mathbb{R}^{n_{i}}, t \geq 0, x_{j}(\cdot) \in L^{\infty}\left(\mathbb{R}_{+}, \mathbb{R}^{n_{j}}\right)$. Note that we treat $x_{j}$ here as an independent measurable input. That is, we neglect that in the formulation of the original problem $x_{j}$ is also given as the solution of a differential equation.

Given two coupled ISS systems of the form (II.6) with the assumption (II.12) it is not possible to conclude asymptotic stability of (II.7) at $x^{*}=0$. However, in this context small gain results are available, by which it is possible to conclude stability.

\section{THE AUXILIARY SYSTEM}

We now assume two as given $\mathscr{K}_{\infty}$-functions $\gamma_{1}, \gamma_{2} \in \mathscr{K}_{\infty}$ that are each locally Lipschitz continuous on $(0, \infty)$. Associated to the systems (II.6) and the defining right hand sides $f_{i}$ we consider the auxiliary functions for $i=1,2$

$$
\begin{aligned}
\tilde{f}_{i, \gamma} & : \mathbb{R}^{n_{i}} \times B(0,1) \rightarrow \mathbb{R}^{n_{i}} \\
\left(x_{i}, u\right) & \mapsto f\left(x_{i}, \gamma_{i}\left(\left\|x_{i}\right\|\right) u\right)
\end{aligned}
$$

Here $B(0,1)$ denotes the unit ball in $\mathbb{R}^{n_{j}}$. (To avoid cumbersome notation we do not refer to the dimension $n_{j}$ when writing this ball.) 
The auxiliary system is now the control system given by

$$
\dot{x}_{i}=f_{i}\left(x_{i}, \gamma_{i}\left(\left\|x_{i}\right\|\right) u\right):=\tilde{f}_{i, \gamma}\left(x_{i}, u\right) .
$$

where $x_{i}^{0} \in \mathbb{R}^{n_{i}}, u \in B_{1}(0) \subset \mathbb{R}^{n_{j}}$.

To explain the significance of the auxiliary system assume for the moment that we have a robust Lyapunov function $V_{i}$ for (III.1), that is, a Lyapunov function such that for all $u \in B(0,1)$ we have

$$
\nabla V_{i}\left(x_{i}\right) f_{i}\left(x_{i}, u\right) \leq-\alpha_{i}\left(\left\|x_{i}\right\|\right),
$$

for some positive definite function $\alpha_{i}$. Then we have the following implication for all $x_{i} \in \mathbb{R}^{n_{i}}, v \in \mathbb{R}^{n_{j}}$.

$$
\begin{array}{r}
\gamma_{i}\left(\left\|x_{i}\right\|\right) \geq\|v\| \Rightarrow \tilde{v}:=\frac{1}{\gamma_{i}\left(\left\|x_{i}\right\|\right)} v \in B(0,1) \\
\Rightarrow \nabla V_{i}\left(x_{i}\right) f_{i}\left(x_{i}, \gamma_{i}\left(\left\|x_{i}\right\|\right) \tilde{v}\right) \leq-\alpha_{i}\left(\left\|x_{i}\right\|\right)
\end{array}
$$

or equivalently

$$
\left\|x_{i}\right\| \geq \gamma_{i}^{-1}(\|v\|) \Rightarrow \nabla V_{i}\left(x_{i}\right) f_{i}\left(x_{i}, v\right) \leq-\alpha_{i}\left(\left\|x_{i}\right\|\right) .
$$

Thus we obtain the following result.

Lemma 2 Let $\gamma \in \mathscr{K}_{\infty}$ be locally Lipschitz on $(0, \infty)$. Consider a subsystem in (II.6) and the auxiliary system (III.1) corresponding to $\gamma$. Then if $V$ is a robust Lyapunov function for (III.1) it is an ISS Lyapunov function for the subsystem of (II.6) with Lyapunov gain $\gamma^{-1}$.

It is clear that this result extends in a straightforward manner to local Lyapunov and ISS Lyapunov functions. As we have seen the problem of computing an ISS Lyapunov function can be reduced to computing a robust Lyapunov function. We thus now study this problem.

\section{THE DOMAIN OF ATTRACTION OF THE AUXILIARY SYSTEM}

In this section we study the properties of one of the subsystems in (III.1). Thus we consider a Lipschitz continuous map $f: \mathbb{R}^{n} \times \mathbb{R}^{m} \rightarrow \mathbb{R}^{n}$, which serves as a representative of one of the maps $f_{i}$ defining the subsystems in (II.6). We now consider the perturbed system

$$
\dot{x}=f_{\gamma}(x, u):=f(x, \gamma(\|x\|) u)
$$

where $x \in \mathbb{R}^{n}, u \in B(0,1) \subset \mathbb{R}^{m}$ and $\gamma \in \mathscr{K}_{\infty} \cup\{0\}$ is locally Lipschitz continuous on $(0, \infty)$. Under these assumptions local Lipschitz continuity of $f_{\gamma}$ is guaranteed on $\mathbb{R}^{n} \backslash\{0\}$, which is sufficient as we assume uniform asymptotic stability in $x^{*}=0$. We denote by $\mathscr{U}:=\left\{u \in L^{\infty}\left(\mathbb{R}, \mathbb{R}^{m}\right) \mid\|u\|_{\infty} \leq 1\right\}$. The solutions of (IV.1) are denoted by $\varphi_{\gamma}\left(\cdot, x^{0}, u\right)$.

We assume that $x^{*}=0$ is uniformly locally asymptotically stable for system (IV.1), i.e.

there exists a constant $r>0$

and a function $\beta$ of class $\mathscr{K} \mathscr{L}$ such that

(H1) $\left\|\varphi_{\gamma}\left(t, x^{0}, u\right)\right\| \leq \beta\left(\left\|x^{0}\right\|, t\right)$ for any $x^{0} \in B(0, r)$, any $u \in \mathscr{U}$, and all $t \geq 0$.
By Sontag's $\mathscr{K} \mathscr{L}$ lemma (see [14]) for any $\beta \in \mathscr{K} \mathscr{L}$ there exist two functions $\alpha_{1}, \alpha_{2} \in \mathscr{K}^{\infty}$ such that

$$
\beta(r, t) \leq \alpha_{2}\left(\alpha_{1}(r) e^{-t}\right) .
$$

In the sequel we will work primarily with the functions $\alpha_{1}, \alpha_{2} \in \mathscr{K}^{\infty}$.

Under the assumption of uniform asymptotical stability of the origin $x^{*}=0$ for (IV.1) $\gamma$, we define the corresponding robust domain of attraction as follows.

Definition 3 We define the (uniform) robust domain of attraction as

$$
\mathscr{D}_{\gamma}=\left\{\begin{array}{ll} 
& \text { there exists } \\
x^{0} \in \mathbb{R}^{n}: & \begin{array}{l}
\eta \in \mathscr{L} \text { such that } \\
\left\|\varphi_{\gamma}\left(t, x^{0}, u\right)\right\| \leq \eta(t) \\
\text { for all } t>0, u \in \mathscr{U}
\end{array}
\end{array}\right\} .
$$

The following properties of $\mathscr{D}_{\gamma}$ are shown in [2].

Proposition 4 Consider system (IV.1) and assume (H1), then

(i) $\mathscr{D}_{\gamma}$ is an open, connected, invariant set with $\operatorname{cl} B(0, r) \subset \mathscr{D}_{\gamma}$.

(ii) $\sup _{u \in \mathscr{U}}\{t(x, u)\} \rightarrow+\infty$ for $x \rightarrow x^{0} \in \partial \mathscr{D}_{\gamma}$ or $\|x\| \rightarrow$ $\infty$, where $t(x, u)=\inf \left\{t>0: \varphi_{\gamma}(t, x, u) \in B(0, r)\right\}$.

(iii) $\mathrm{cl} \mathscr{D}_{\gamma}$ is an invariant set which is contractible to 0.

(iv) If for some $u_{0} \in U f_{\gamma}\left(\cdot, u_{0}\right)$ is of class $C^{1}$, then $\mathscr{D}_{\gamma}$ is $C^{1}$-diffeomorphic to $\mathbb{R}^{n}$.

It is shown in [1], [2], [3] how a Zubov type equation may be formulated that allows for the computation of a maximal Lyapunov function on the domain of attraction. In this paper we use the formulation of [3], which is a slight generalization of [2]. To this end an optimal control problem is defined using a running cost $g$, which is chosen in such a manner that the function $g: \mathbb{R}^{n} \times U \rightarrow \mathbb{R}$ is continuous and satisfies

(i) For all $u \in U, g(x, u) \leq C \alpha_{2}^{-1}(\|x\|)$ for all $x \in \mathbb{R}^{n}, \alpha_{2}$ from (IV.2) and some $C>0$, and $g(x, u)>0$ for $x \neq 0$.

(H2) (ii) There exists a constant $g_{0}>0$ such that $\inf \{g(x, u) \mid x \notin B(0, r), u \in U\} \geq g_{0}$.

(iii) For each $R>0$ there exists $L_{R}>0$ such that $\|g(x, u)-g(y, u)\| \leq L_{R}\|x-y\|$ for all $\|x\|,\|y\| \leq R$, and all $u \in U$.

We now introduce the value function of a suitable optimal control problem related to (IV.1). Consider the following nonnegative, extended value functional $J: \mathbb{R}^{n} \times \mathscr{U} \rightarrow \mathbb{R} \cup$ $\{+\infty\}$

$$
J_{\gamma}(x, u):=\int_{0}^{+\infty} g\left(\varphi_{\gamma}(t), u(t)\right) d t,
$$

and the optimal value function

$$
v_{\gamma}(x):=\sup _{u \in \mathscr{U}} 1-e^{-J_{\gamma}(x, u)} .
$$


Since $g$ is nonnegative it is immediate that $v(x) \in[0,1]$ for all $x \in \mathbb{R}^{n}$. Furthermore, standard techniques from optimal control imply that $v_{\gamma}$ satisfies a dynamic programming principle, i.e. for each $t>0$ we have

$$
v_{\gamma}(x)=\sup _{u \in \mathscr{U}}\left\{(1-G(x, t, u))+G(x, t, u) v_{\gamma}(x(t, x, u))\right\}
$$

with

$$
G(t, x, u):=\exp \left(-\int_{0}^{t} g(x(\tau, x, u), u(\tau)) d \tau\right) .
$$

A simple application of the chain rule shows (1$G(x, t, u))=\int_{0}^{t} G(x, \tau, u) g(x(\tau, x, u), u(\tau)) d \tau$ implying

$$
\begin{gathered}
v_{\gamma}(x)=\sup _{u \in \mathscr{U}}\left\{\int_{0}^{t} G(x, \tau, u) g(x(\tau, x, u), u(\tau)) d \tau\right. \\
+G(x, t, u) v(x(t, x, u))\}
\end{gathered}
$$

The next proposition shows the relation between $\mathscr{D}_{\gamma}$ and $v_{\gamma}$, and the continuity of $v_{\gamma}$.

\section{Proposition 5 Assume (H1), (H2). Then}

(i) $v_{\gamma}(x)<1$ if and only if $x \in \mathscr{D}_{\gamma}$.

(ii) $v_{\gamma}(x)=0$ if and only if $x=0$.

(iii) $v_{\gamma}$ is continuous on $\mathbb{R}^{n}$.

(iv) $v_{\gamma}(x) \rightarrow 1$ for $x \rightarrow x^{0} \in \partial \mathscr{D}_{\gamma}$ and for $|x| \rightarrow \infty$.

Finally, it follows that $v$ can be characterized as the unique viscosity solution of the Zubov equation

$$
\sup _{\|u\| \leq 1}\left\{D v(x) f_{\gamma}(x, u)+(1-v(x)) g(x, u)\right\}=0
$$

Theorem 6 Consider the system (IV.1) and a function $g$ : $\mathbb{R}^{n} \times A \rightarrow \mathbb{R}$ such that (H1) and (H2) are satisfied. Then (IV.7) has a unique bounded and continuous viscosity solution $v$ on $\mathbb{R}^{n}$ satisfying $v(x)=0$ for $x=0$.

This function coincides with $v_{\gamma}$ from (IV.3). In particular the characterization $\mathscr{D}_{\gamma}=\left\{x \in \mathbb{R}^{n} \mid v_{\gamma}(x)<1\right\}$ holds.

Proposition 7 Assume $(\mathrm{H} 1)$ and $(\mathrm{H} 2)$ and consider the unique viscosity solution $v_{\gamma}$ of (IV.7) with $v_{\gamma}(0)=0$. Then the function $v_{\gamma}$ is a robust Lyapunov function for the system (IV.1) on $\mathscr{D}_{\gamma}$. More precisely we have

$$
\begin{aligned}
& v_{\gamma}\left(x\left(t, x^{0}, u\right)\right)-v_{\gamma}\left(x^{0}\right) \\
& \leq\left[1-e^{-\int_{0}^{t} g(x(\tau), u(\tau)) d \tau}\right]\left(v_{\gamma}\left(x\left(t, x^{0}, u\right)\right)-1\right)<0
\end{aligned}
$$

for all $x^{0} \in \mathscr{D}_{\gamma} \backslash\{0\}$ and all functions $u \in \mathscr{U}$.

Now we turn to the Lipschitz property.

Proposition 8 Assume (H1) and (H2) and consider the unique viscosity solution $v_{\gamma}$ of (IV.7) with $v_{\gamma}(x)=0$ for all $x \in D$.

If $f_{\gamma}(\cdot, u)$ and $g(\cdot, u)$ are uniformly Lipschitz continuous in $\mathscr{D} \gamma$, with constants $L_{f}, L_{g}>0$ uniformly in $u \in U$, and if there exists an open neighborhood $N$ of 0 such that for all $x, y \in N$ the inequality

$$
\begin{aligned}
& |g(x, u)-g(y, u)| \\
& \leq K \alpha_{2}^{-1}(\max \{\|x\|,\|y\|\})^{s}\|x-y\|
\end{aligned}
$$

holds for some $K>0, s>L_{f}$ and $\alpha_{2}$ from (IV.2), then the function $v_{\gamma}$ is Lipschitz continuous in $\mathbb{R}^{n}$ for all $g$ with $g_{0}>0$ from (H2) sufficiently large.

Finally, we note that the Lyapunov function $v_{\gamma}$ may be interpreted as a local ISS-Lyapunov function on the set $D_{\gamma}$ for the system

$$
\dot{x}=f(x, u) \text {. }
$$

Proposition 9 Consider system (IV.8). The function $v_{\gamma}$ defined as the unique viscosity solution of (IV.7) is a local ISS-Lyapunov function on $\mathscr{D}_{\gamma}$ in the sense that if $x \in \mathscr{D}_{\gamma}$ then

$$
\begin{array}{r}
\|x\| \geq \gamma^{-1}(\|u\|) \quad \Rightarrow \\
v_{\gamma} \text { is a viscosity subsolution of } \\
\nabla v_{\gamma} f_{\gamma}(x, u) \leq-\left(1-v_{\gamma}(x)\right) g(x, u) .
\end{array}
$$

Proof: This may be seen as in the proof of Lemma 2. The treatment in the framework of viscosity solutions poses no extra difficulty.

The formulation in (IV.9) is a viscosity formulation of the implication form for ISS Lyapunov functions. The important point here, is that it implies the desired decay properties as in (II.4).

We also note that the following properties of $\mathscr{D}_{\gamma}$ in dependence of $\gamma$. Note that $\mathscr{D}_{0}$ corresponds to the choice $\gamma \equiv 0$.

Proposition 10 Let $\gamma_{1} \leq \gamma_{2} \in \mathscr{K}_{\infty}$ be locally Lipschitz on $(0, \infty)$, then $\mathscr{D}_{\gamma_{1}} \supset \mathscr{D}_{\gamma_{2}}$. In particular, $\mathscr{D}_{\gamma} \subset \mathscr{D}_{0}$ for all $\gamma \in \mathscr{K}_{\infty}$.

Proof: $\quad$ This follows immediately from $f\left(x, \gamma_{1}(\|x\|) B(0,1)\right) \subset f\left(x, \gamma_{2}(\|x\|) B(0,1)\right)$ for all $x \in \mathbb{R}^{n}$ if $\gamma_{1} \leq \gamma_{2}$

In particular, the previous result shows a drawback of our approach. For our coupled system the best we can hope for as an estimate for the domain of attraction of the coupled system will be the set $\mathscr{D}_{\gamma, 1} \times \mathscr{D}_{\gamma, 2}$ because outside of these sets we have no information about the decay of the subsystems.

\section{Coupled systems}

We now want to choose two gain functions $\gamma_{1}^{-1}, \gamma_{2}^{-1}$ for the individual subsystems system, so that a Lyapunov function can be obtained for the coupled system. To this end we need the following small gain result. Given the Lyapunov functions $v_{1}, v_{2}$ with gains $\gamma_{12}^{-1}, \gamma_{21}^{-1}$ we consider the matrix

$$
\Gamma:=\left(\begin{array}{cc}
0 & \gamma_{12}^{-1} \\
\gamma_{21}^{-1} & 0
\end{array}\right) \text {. }
$$


The matrix $\Gamma$ may be interpreted as an operator on the positive orthant $\mathbb{R}_{+}^{2}$ which acts through $s=\left(s_{1}, s_{2}\right) \mapsto \Gamma(s):=$ $\left(\gamma_{12}^{-1}\left(s_{2}\right), \gamma_{21}^{-1}\left(s_{1}\right)\right)$. We also define a diagonal operator $E$ : $\mathbb{R}_{+}^{2} \rightarrow \mathbb{R}_{+}^{2}$ which is defined through two $\mathscr{K}_{\infty}$ functions $\eta_{1}, \eta_{2}$ and defined by $E(s):=\left(\left(\mathrm{id}+\eta_{1}\right)\left(s_{1}\right),\left(\mathrm{id}+\eta_{2}\right)\left(s_{2}\right)\right)$. The following result is a special case of the general small gain theorem obtained in [4], [7], [12]. In this form it is quite similar to the result from [10] which is obtained for a different formulation of the ISS condition.

For the small gain result we need a deviation from the formulation in (II.4). Namely, we assume that each subsystem $i=1,2$ has an ISS Lyapunov function satisfying for $i=1,2$ that there exist $\psi_{1, i}, \psi_{2, i} \in \mathscr{K}_{\infty}$ and $\chi, \alpha \in \mathbb{K}$ with

$$
\begin{gathered}
\psi_{1, i}(\|x\|) \leq V_{i}(x) \leq \psi_{2, i}(\|x\|), x \in \mathbb{R}^{n} \\
V_{i}\left(x_{i}\right) \geq \chi_{i j}\left(V_{j}\left(x_{j}\right)\right) \Rightarrow \nabla V_{i}(x) f_{i}\left(x_{i}, x_{j}\right) \leq-\alpha_{i}\left(V_{i}\left(x_{i}\right)\right) .
\end{gathered}
$$

Also note that because of (V.1) the implication (V.2) is valid for $i=1,2$, if for $i \neq j$ we have

$$
\begin{gathered}
\left\|x_{i}\right\| \geq \psi_{2, i}^{-1} \circ \chi_{i} \circ \psi_{1, j}\left(\left\|x_{j}\right\|\right) \Rightarrow \\
\nabla V_{i}(x) f_{i}\left(x_{i}, x_{j}\right) \leq-\alpha_{i}\left(V_{i}\left(x_{i}\right)\right) .
\end{gathered}
$$

Thus the natural gains we have to consider after constructing a Lyapunov function which has a gain $\gamma_{i}^{-1}$ as in (IV.9) is given by $\tilde{\gamma}_{i}:=\psi_{2, i} \circ \gamma_{i}^{-1} \circ \psi_{1, j}^{-1}$.

Using the formulation in (V.2) we can state the stability result for the feedback interconnection. In the statement we use the order on $\mathbb{R}_{+}^{2}$ given by $a \leq b: \Leftrightarrow a_{i} \leq b_{i}, i=1,2$. Thus the negation $a \not \leq b$ means that $a_{i}>b_{i}$ for some $i$.

Theorem 11 Consider the coupled system (II.6) and assume that for each of the subsystems there exists an ISS Lyapunov function $V_{i}, i=1,2$ in the sense of (V.2) with Lyapunov gain $\gamma_{i j}^{-1}, i, j=1,2, i \neq j$. If there exists a diagonal operator $E$ such that the small gain condition

$$
E \circ \Gamma(s) \nsupseteq s, \forall s \in \mathbb{R}_{+}^{2} \backslash\{0\},
$$

is satisfied, then there exists a continuously differentiable path $\sigma:[0, \infty) \rightarrow \mathbb{R}^{2}$, such that $\sigma^{\prime}$ is bounded and so that

$$
E \circ \Gamma(\sigma(\tau))<\sigma(\tau), \quad \forall \tau \in(0, \infty) .
$$

A Lyapunov function for the coupled system is then given by

$$
V\left(x_{1}, x_{2}\right):=\max \left\{\sigma_{1}^{-1}\left(V_{1}\left(x_{1}\right)\right), \sigma_{2}^{-1}\left(V_{2}\left(x_{2}\right)\right)\right\} .
$$

In this formulation $\sigma_{i}$ denotes the $i$-th component function of the path $\sigma$. Also note that in the case of two systems in feedback interconnection (V.4) is equivalent to the existence of $\eta_{1}, \eta_{2}$ such that

$$
\left(\mathrm{id}+\eta_{1}\right) \circ \gamma_{12}^{-1} \circ\left(\mathrm{id}+\eta_{2}\right) \circ \gamma_{21}^{-1}(\tau)<\tau, \quad \forall \tau>0 .
$$

A local version of this result has been obtained in [6].

For each of these gain functions we can compute $\mathscr{D}_{\gamma_{i}}$ and $v_{\gamma_{i}}$ by solving the corresponding Zubov equation (IV.7). We now use these functions to obtain an estimate for the domain of attraction of the coupled system.
Since we only have local version of the Lyapunov functions we need a local version suitable for our case. The procedure we now propose is the following:

(i) For each of the subsystems $i=1,2$ choose $\gamma_{i} \in \mathscr{K}_{\infty}$ and compute the maximal Lyapunov function $v_{i}$ on $D_{\gamma, i}$ by solving the corresponding Zubov equation (IV.7).

(ii) For each $v_{\gamma, i}$ compute a function $\psi_{i, 1}$ such that the left hand side of (V.1) is satisfied.

(iii) The gain for each of the Lyapunov functions is then by (II.5) given by

$$
\tilde{\gamma}_{i j}:=\psi_{2, i} \circ \gamma_{i}^{-1} \circ \psi_{1, j}^{-1}
$$

(iv) Check that the two gains $\tilde{\gamma}_{12}, \tilde{\gamma}_{21}$ satisfy the small gain condition (V.4).

(v) If this is the case choose the path $\sigma$ along which $E \circ \Gamma$ is decreasing as in (V.5). ${ }^{1}$

(vi) Define

$$
V\left(x_{1}, x_{2}\right):=\max \left\{\sigma_{1}^{-1}\left(v_{1}\left(x_{1}\right)\right), \sigma_{2}^{-1}\left(v_{2}\left(x_{2}\right)\right)\right\},
$$

and let $\rho:=\min \left\{\sigma_{1}^{-1}(1), \sigma_{2}^{-1}(1)\right\}$.

We claim that provided all steps in the construction can be completed successfully then with this choice of $V$ we have that $V^{-1}([0, \rho))$ is a subset of the domain of attraction of the coupled system. This is the gist of the following theorem.

Theorem 12 Consider the coupled system (II.6). Assume for each of the subsystems a solution $v_{i}$ of the Zubov equation (IV.7) is available. Assume furthermore, that the gains defined in (V.6) satisfy the small gain condition (V.4). Then for the function $V$ defined in (V.7) is a local viscosity Lyapunov function for the coupled system and we have $\mathscr{D}_{V}:=V^{-1}([0, \rho) \subset \mathscr{D}$ from (II.8).

Proof: Without loss of generality we may assume that $\rho=1$, because we can always rescale the path $\sigma$. We first note, that the choice of $\rho$ ensures, that $V\left(x_{1} . x_{2}\right)<$ $\rho=1$ implies that $x_{1} \in D_{\gamma, 1}$ and $x_{2} \in D_{\gamma, 2}$, because of Proposition 5 (i).

Let $x \in \mathscr{D}_{V} \backslash\{0\}$. We assume first that for a given $x=$ $\left(x_{1}, x_{2}\right)$ we have $V\left(x_{1}, x_{2}\right)=\sigma^{-1}\left(v_{1}\left(x_{1}\right)\right)>\sigma_{2}^{-1}\left(v_{2}\left(x_{2}\right)\right)$. Then it follows that $v_{1}\left(x_{1}\right)>\sigma_{1} \circ \sigma_{2}^{-1}\left(v_{2}\left(x_{2}\right)\right)$ and by the definition of $\sigma$ this implies $v_{1}\left(x_{1}\right)>\tilde{\gamma}_{12}\left(v_{2}\left(x_{2}\right)\right)$. As $x_{1} \in \mathscr{D}_{\gamma, 1}$ this implies that $\nabla V\left(x_{1}, x_{2}\right)=\nabla v_{i}\left(x_{1}\right)<-(1-$ $\left.v_{1}\left(x_{1}\right)\right) g\left(x_{1}, x_{2}\right)$ in the viscosity sense. The same argument applies vice versa if $V\left(x_{1}, x_{2}\right)=\sigma_{2}^{-1}\left(v_{2}\left(x_{2}\right)\right)>\sigma^{-1}\left(v_{1}\left(x_{1}\right)\right)$. This shows that the decay condition holds almost everywhere on $\mathscr{D}_{V}$. This implies the assertion.

\section{EXAMPLE}

In this section we present a very simple example which explains the steps that have to be undertaken in the construction. Consider the coupled system

$$
\begin{aligned}
& \dot{x}_{1}=-x_{1}+c_{12} x_{2} \\
& \dot{x}_{2}=-x_{2}+c_{21} x_{1} .
\end{aligned}
$$

\footnotetext{
${ }^{1}$ This can be done numerically in a simple manner, see [12]
} 
Each of the subsystems is globally ISS with ISS-Lyapunov function $V_{i}\left(x_{i}\right)=\left|x_{i}\right|$. With this choice we obtain for any $\varepsilon>0$ and $i \neq j, i, j \in\{1,2\}$ the implication condition

$$
\left|x_{i}\right|>\left(c_{i j}+\varepsilon\right)\left|x_{j}\right| \Rightarrow \dot{V}_{i}\left(x_{i}\right) \leq-\frac{\varepsilon}{c_{i j}+\varepsilon}\left|x_{i}\right|
$$

Hence for any $\varepsilon>0$ the gain can be chosen to be $\gamma_{i j}:=$ $\left(c_{i j}+\varepsilon\right)$. The Lyapunov function that would be constructed by Zubov's method is of the form $v(x):=1-e^{-V_{i}(x)}$ and it can be readily checked, that the same implication condition is valid in this case.

By the small gain condition we have to require that $\gamma_{12} \gamma_{21}<1$. By choosing $\varepsilon>0$ small enough this is possible whenever $c_{12} c_{21}<1$ and it is easy to see that in this simple case, this is indeed necessary and sufficient for stability of the coupled system.

Now to construct the overall Lyapunov function we need to construct the path $\sigma$ and in our case it is easy to see, that for $\sigma: t \mapsto\left(2 t,\left(\gamma_{21}+\gamma_{12}^{-1}\right) t\right)$ we have

$$
\left[\begin{array}{cc}
0 & \gamma_{12} \\
\gamma_{21} & 0
\end{array}\right] \sigma(t)=\left[\begin{array}{c}
\left(1+\gamma_{12} \gamma_{21}\right) t \\
2 \gamma_{21} t
\end{array}\right]<\sigma(t) .
$$

The existence of the diagonal operator $E$ is automatic in this case, because of the linearity of the gains. Hence an overall Lyapunov function is obtained by setting

$$
V\left(x_{1}, x_{2}\right):=\max \left\{\frac{1}{2} v\left(x_{1}\right), \frac{1}{\left(\gamma_{21}+\gamma_{12}^{-1}\right)} v\left(x_{2}\right)\right\} .
$$

As expected the method underestimates the actual domain of attraction of the coupled system.

\section{STATE TRANSFORMATION}

In many coupled systems it is not the case, that each of the subsystems is ISS with respect to the inputs from the other subsystems even though the overall system has an asymptotically stable fixed point at $x^{*}=0$. In this section we want to explain briefly what can be done to make the approach of this paper applicable. In a general framework it is possible to apply the ideas from [9], but a discussion of this approach is beyond the scope of this paper.

We assume we are given a system of the form (II.6), which is continuously differentiable at $x^{*}=0$. Denote the Jacobian at 0 by

$$
A:=\left(\begin{array}{ll}
A_{11} & A_{12} \\
A_{21} & A_{22}
\end{array}\right),
$$

where the blocks correspond to the partitioning $x=\left(x_{1}, x_{2}\right)$. We assume that $A$ is Hurwitz, so that we have exponential stability at $x^{*}=0$ for (II.6). In case the small gain condition cannot be satisfied, consider the Lyapunov equation

$$
A^{T} P+P A=-I,
$$

which has a unique positive definite solution $P$. If we apply the congruence transformation $P^{-1 / 2}$ to this equation we obtain

$$
P^{-1 / 2} A^{T} P^{1 / 2}+P^{1 / 2} A P^{-1 / 2}=-P .
$$

This shows that the state transformation $\hat{x}=P^{1 / 2} x$ results in the Jacobian $\hat{A}=P^{1 / 2} A P^{-1 / 2}$ which has the identity as a Lyapunov function. This shows that for the original nonlinear systems at least locally the subsystems satisfy the desired ISS conditions as well as the small gain condition, because this is true for their linearizations. Hence after this transformation the approach of this paper may be applied.

\section{CONCLUSIONS}

In this paper we have outlined a way for the estimation of domains of attractions of coupled systems that satisfy an ISS condition and additionally a small gain condition. For each of the subsystems a robust Lyapunov function is calculated which serves as an ISS Lyapunov function. To this end Zubov's method can be applied. As yet, we have no systematic way of choosing the gains that are needed in step one of this construction. This is the topic of ongoing research.

\section{REFERENCES}

[1] F. Camilli, L. Grüne, and F. Wirth. A regularization of Zubov's equation for robust domains of attraction. In A. Isidori et al., editor, Nonlinear Control in the Year 2000, volume 258 of Lecture Notes in Control and Information Sciences, pages 277-290. Springer-Verlag, Berlin, 2000.

[2] Fabio Camilli, Lars Grüne, and Fabian Wirth. A generalization of Zubov's method to perturbed systems. SIAM J. Control Optimization, 40(2):496-515, 2001.

[3] Fabio Camilli, Lars Grüne, and Fabian Wirth. A generalization of Zubov's method to perturbed systems. In Proc. 41st IEEE Conference on Decision and Control, CDC2002, pages 3518-3523, Las Vegas, NV, US, Dec. 2002. IEEE.

[4] S. Dashkovskiy, B. Rüffer, and F. Wirth. Construction of ISS Lyapunov functions for networks. Berichte aus der Technomathematik 06-06, Zentrum für Technomathematik, Bremen, Germany, 2006.

[5] S. Dashkovskiy, B. Rüffer, and F. Wirth. An ISS small-gain theorem for general networks. Math. Control Signals Systems, 19:93-122, 2007.

[6] S. Dashkovskiy, B. Rüffer, and F. Wirth. Numerical verification of local input-to-state stability for large netwoks. In Proc. of 46th IEEE Conference on Decision and Control, CDC 2007, pages 4471 - 4476, 12-14 December, 2007, New Orleans, USA, December 2007.

[7] S. Dashkovskiy, B. S. Rüffer, and F. R. Wirth. A Lyapunov ISS small gain theorem for strongly connected networks. In Proc. 7th IFAC Symposium on Nonlinear Control Systems, NOLCOS2007, pages 283288, Pretoria, South Africa, August 2007.

[8] Lars Grüne. Input-to-state dynamical stability and its Lyapunov function characterization. IEEE Trans. Automat. Control, 47(9):14991504, 2002.

[9] Lars Grüne, Eduardo D. Sontag, and Fabian R. Wirth. Asymptotic stability equals exponential stability, and ISS equals finite energy gain-if you twist your eyes. Systems Control Lett., 38(2):127-134, 1999.

[10] Z.-P. Jiang, I. M. Y. Mareels, and Y. Wang. A Lyapunov formulation of the nonlinear small-gain theorem for interconnected ISS systems. Automatica J. IFAC, 32(8):1211-1215, 1996.

[11] Z.-P. Jiang, A. R. Teel, and L. Praly. Small-gain theorem for ISS systems and applications. Math. Control Signals Systems, 7:95-120, 1994.

[12] B. S. Rüffer. Monotone Systems, Graphs, and Stability of Large-Scale Interconnected Systems. Dissertation, Fachbereich 3, Mathematik und Informatik, Universität Bremen, Germany, August 2007. Available online: http://nbn-resolving.de/urn:nbn:de:gbv:46-diss000109058.

[13] E. D. Sontag. Smooth stabilization implies coprime factorization. IEEE Trans. Automat. Control, 34(4):435-443, 1989.

[14] E. D. Sontag. Comments on integral variants of ISS. Systems Control Lett., 34:93-100, 1998.

[15] E. D. Sontag and Y. Wang. On characterizations of the input-to-state stability property. Systems Control Lett., 24(5):351-359, 1995. 